\title{
11th International Conference on the Chemistry of Antibiotics and other Bioactive Compounds (ICCA-11) Donostia-San Sebastian, Spain 29 September-2 October 2009
}

\author{
Herbert A Kirst \\ The Journal of Antibiotics (2010) 63, 45-48; doi:10.1038/ja.2009.115; published online 27 November 2009
}

The 11th International Conference on the Chemistry of Antibiotics and Other Bioactive Compounds (ICCA-11) was held in the conference hall of the historic Miramar Royal Residence in DonostiaSan Sebastian, Spain. The conference was very well-organized by Professor Jesus M Aizpurua (Universidad del Pais Vasco) and his organizing committees. In addition to the excellent scientific program, approximately 90 conference attendees enjoyed the perfect autumn weather and spectacular views from Miramar Park overlooking La Concha Bay framed by Mt Igeldo and Mt Urgull at its opposite ends (Figures 1-3). Owing to space limitations, this summary of the conference proceedings will necessarily be very selective and incomplete and will only briefly summarize selected features from the many lectures.

After an evening opening reception, the conference began with a plenary lecture by Professor Andrew Myers (Harvard University) who described his research on the total synthesis of tetracycline antibiotics. ${ }^{1}$ The four synthetic routes developed thus far have transformed the preparation of tetracycline derivatives from a semi-synthetic strategy to a totally synthetic endeavor that has allowed efficient syntheses of derivatives, including many previously unattainable at less reactive sites. This work led to the creation of Tetraphase Pharmaceuticals, resulting in a very rapid rise in the number of compounds being synthesized, with over 1500 prepared by August, 2009. A lead compound is in Phase I trial for intravenous administration with other candidates (oral; broad spectrum) expected to follow.

In other plenary lectures, Professor Ernest Giralt (University of Barcelona) discussed the protein network and protein-protein interactions as potential drug targets for design of small ligands that bind and modify these interactions. He discussed applications to design and prepare ligands that would complement 'holes' or untangle 'knots' that might appear in a folded protein structure to restore protein function. ${ }^{2}$ Professor Yujiro Hayashi (Tokyo University of Science) described his work on asymmetric synthesis using novel organocatalysts, especially ones based on proline and prolinol. Asymmetric reactions were applied to the total synthesis of several complex natural products, such as the ansamycin-related cytotrienin A and the important antiviral agent, tamiflu. ${ }^{3}$ Professor Ferenc Fülöp (University of Szeged) emphasized the importance of university-industrial networks to open collaborations and create synergistic research partnerships. He then reported his research on the synthesis of cyclic $\beta$-amino acids, where such structures fit the criteria for potential new drugs, as exemplified by the antifungal agent, icofungipen, now in Phase III trial. These amino acids are also useful in programs for building novel peptide foldamers (non-natural folded polymers). ${ }^{4}$

Dr Ada Yonath (Weizmann Institute) presented a colorful and highly detailed 40-min video depicting the structural complexities of the ribosome, the mechanism by which proteins are synthesized, how that process is disrupted by the binding of certain antibiotics and the ways in which microbial resistance and cross-resistance to antibiotics can develop in the ribosome. ${ }^{5,6}$ The following week, Dr Yonath was a recipient of the Nobel Prize in Chemistry for this pioneering work and its applications to the design of new antibiotics. Congratulations to $\mathrm{Dr}$ Yonath on this outstanding achievement.

One conference theme concerned discovery processes for new natural product antibiotics and how traditional methods could be modified and modernized to overcome obstacles, such as the low probability of finding new molecules, the time and resources devoted to reducing the unwanted re-discovery of known and previously discarded structures and the need to improve pharmaceutical features of drug candidates beyond in vitro potency and spectrum of activity. ${ }^{7-9}$ Professor Roderich Süssmuth (Technische University of Berlin) described his drug-oriented biological chemistry research using chemical or bioassay-guided screening or genomic analysis of producing microbes to find lead structures, such as abyssomicin, proximicin and labyrinthopeptin $\mathrm{A} 1$ and $\mathrm{A} 2$. He also described microbial engineering strategies in which biosynthetic pathways can be redirected by processes such as combinatorial biosynthesis or feeding unnatural substrates to create new structures, as exemplified by emodepside (analog of the anthelmintic PF1022). Dr Andres 
Francesch (PharmaMar, Madrid, Spain) reviewed the marine focus of his organization as the source for finding new active natural products from its library of more than 74000 marine samples. He also described the successful synthesis and development of Yondelis (trabectedin) for treatment of soft tissue sarcoma and four other compounds in early trials (Aplidin and Irvalec in Phase II, Zalypsis and PM01183 in Phase I).

Dr Sheo Singh (Merck, Rahway, NJ, USA) reported a research program on the thiazole family of peptide antibiotics, particularly thiazomycin, in an effort to overcome undesirable drug features such as poor water solubility. Over 300 derivatives with good bactericidal activity were prepared and a pre-clinical candidate was identified, but problems with development of microbial resistance resulted in cessation of this program. Professor Andreas Kirschning (Leibniz University of Hannover) focused on mutasynthesis as a strategy to complement semi-synthesis and total synthesis for obtaining new natural products having improved features compared with the parent molecule. Mutants of ansamycin-producing strains (ansamitocin and geldanamycin) that were biosynthetically blocked in amino-hydroxybenzoic acid were used to incorporate unnatural starting units (mutasynthons), and several post-PKS mutant strains have also been obtained.

Another major theme of the conference was chemical synthesis to explore structure-activity relationships and produce new drug candidates. ${ }^{9,10}$ Professor Fredrik Almqvist (Umeå University) focused on inhibiting bacterial virulence as a target for new antibiotics. He used bicyclic 2-pyridones as a chemical scaffold to synthesize agents that inhibited formation of pili and curli in bacteria, resulting in certain bald bacterial strains showing reduced virulence. Dr Tomoyasu Hirose (Kitasato University) reported the use of click chemistry to synthesize more potent triazole analogs of the macrolide antibiotic, spiramycin I and the inhibitor of bacterial chitinase $\mathrm{B}$, argifin.

Professor Pal Herczegh (University of Debrecen) described converting the terminal amine of ristocetin aglycone and teicoplanin pseudoaglycone to an azide that was then coupled via click chemistry with lipid- or carbohydrate-linked acetylenes to produce new triazole glycopeptide derivatives. Some of these new derivatives showed interesting activity against Gram-positive bacteria and also influenza viruses. Click chemistry was a very popular transformation employed by several other speakers. It was used by Dr Loránd Kiss (University of Szeged) to prepare several new triazole derivatives of carbanucleosides and by Itxaso Azcune (University of Basque Country) to synthesize sugar-triazole- $\beta$-lactam compounds as inhibitors of lectin. Click chemistry was also used by Ainara Garcia-Gallastegi (INASMET) as the functionality to prepare vancomycin-linked carbon nanotubes for antibiotic immobilization. Dr Daisuke Matsuda (Kitasato University) reported studies on the derivatization of the ACAT inhibitor, pyripyropene A, that had resulted in three new derivatives showing greater activity when tested in their atherogenic mouse model of cardiovascular disease.

A third major conference topic was the exploration of useful new drug targets. ${ }^{11,12}$ Professor Markus Kalesse (Leibniz University of Hannover) discussed work on the anti-tumor agents, disorazole and argyrin A and F. Argyrin A is a cyclic peptide obtained from a myxobacterium that was found from screening 60000 samples for compounds that stabilize P27 and prevent its cellular degradation. Such stabilization is desirable as P27 is a cyclin kinase inhibitor that functions as a tumor suppressor, which is eliminated from cancer cells, thereby removing the suppression of growth. Argyrin was described as more active than velcade and thus represents a potential new natural product anti-tumor agent. Dr Stephan Sieber (LMU München) described their search for new antimicrobial cell targets by preparing chemical probes from small $\beta$-lactones and $\beta$-lactams containing a terminal acetylene function suitable for click chemistry to attach a visualization tag. After lysis of the probe-labeled proteome followed by the click chemistry, fluorescent SDS-gel electrophoresis and MS analysis were used to identify those enzymes selectively targeted by

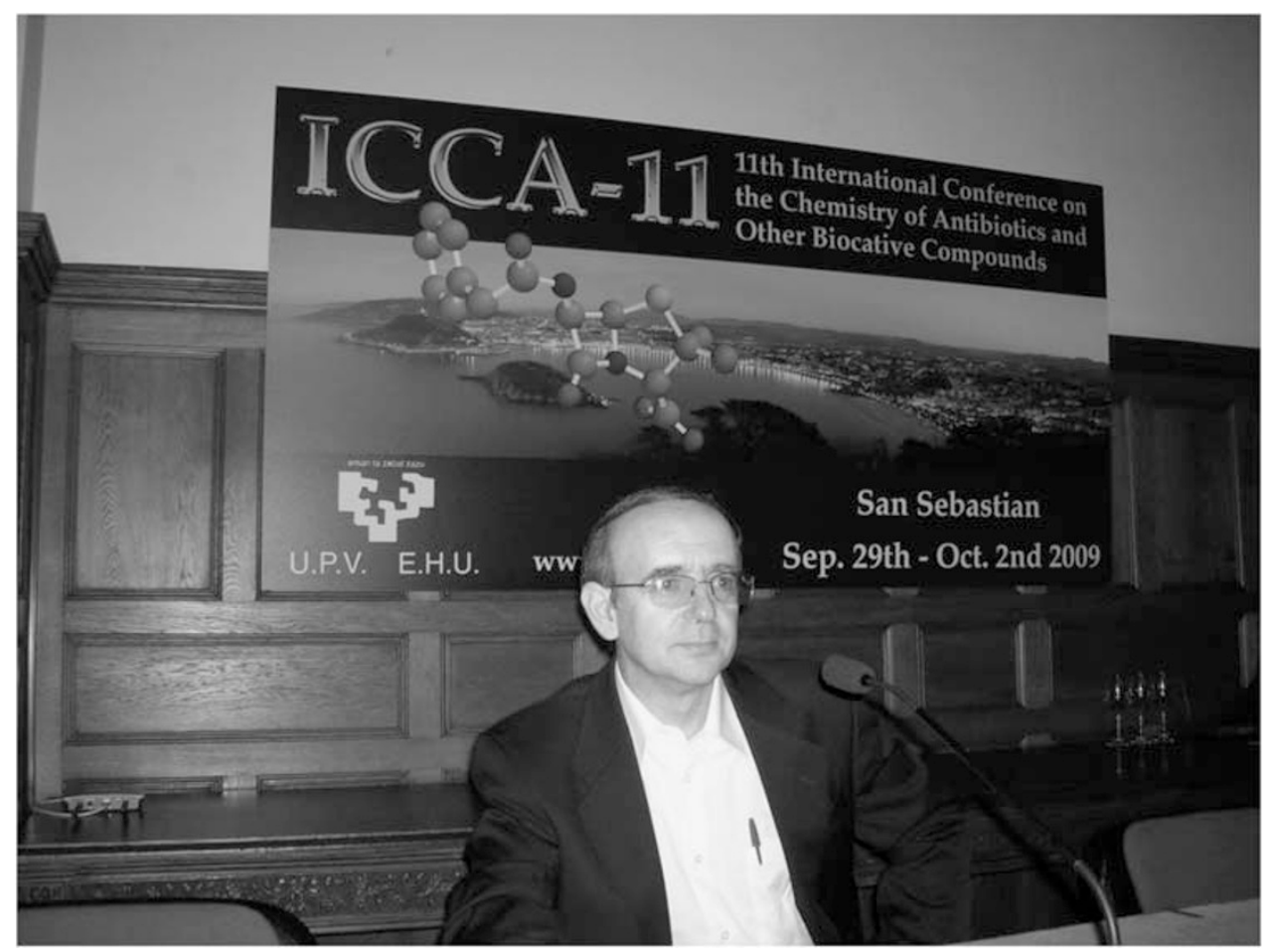

Figure 1 Professor Jesus Aizpurua, the conference organizer, at the front of the lecture hall. 
the probes. An ATP-dependent Clp protease was found to be a global regulator of virulence.

Professor Cesare Gennari (University of Milan) synthesized cyclic RGD peptidomimetic structures containing an embedded diketopi- perazine moiety as ligands for binding to integrins. NMR and computational studies were used to predict conformations and binding of the compounds. Professor Fernando Cossio (University of Basque Country) designed and prepared a group of unnatural nitro-

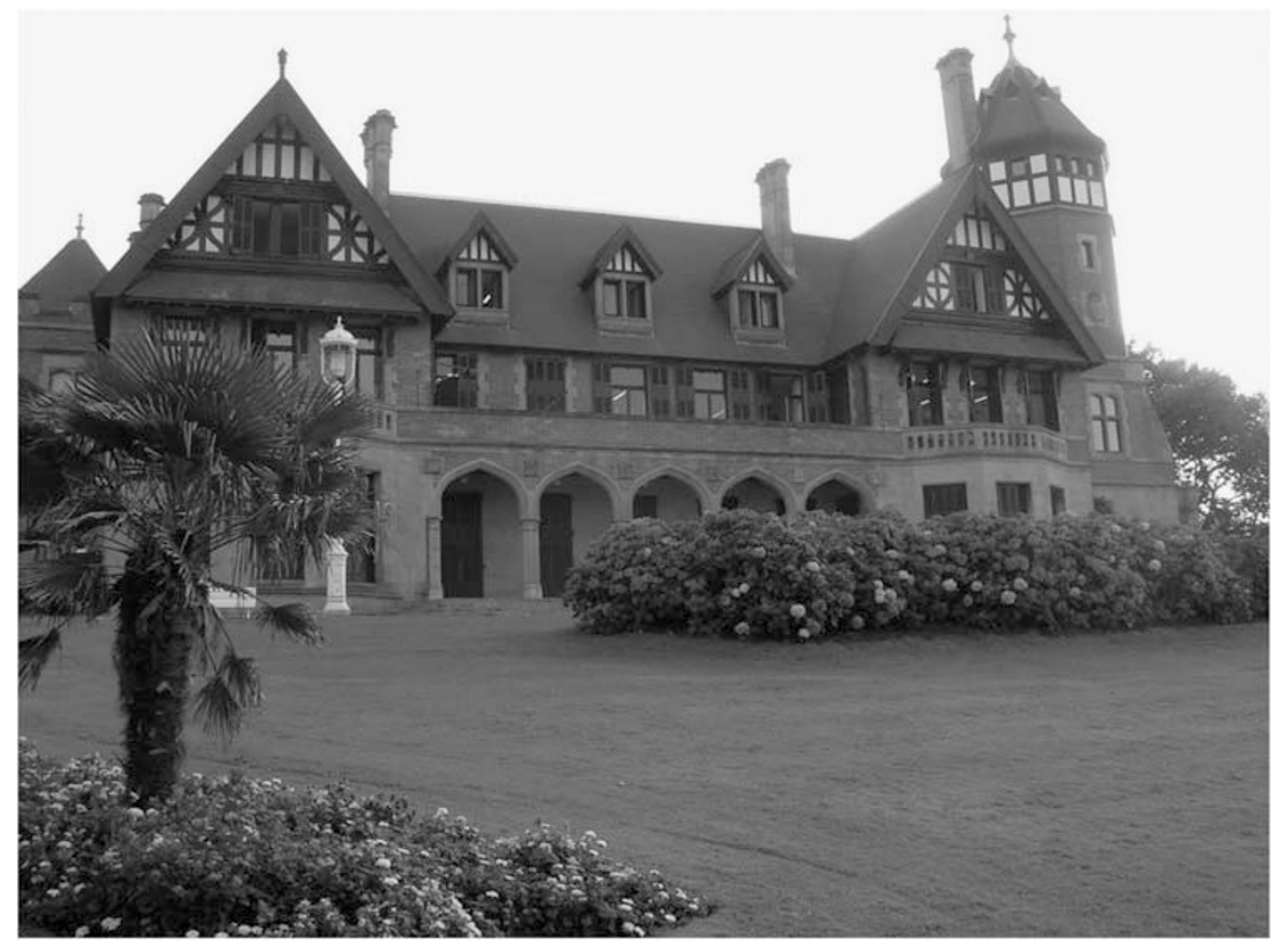

Figure 2 The Miramar Royal Residence, site of ICCA-11.

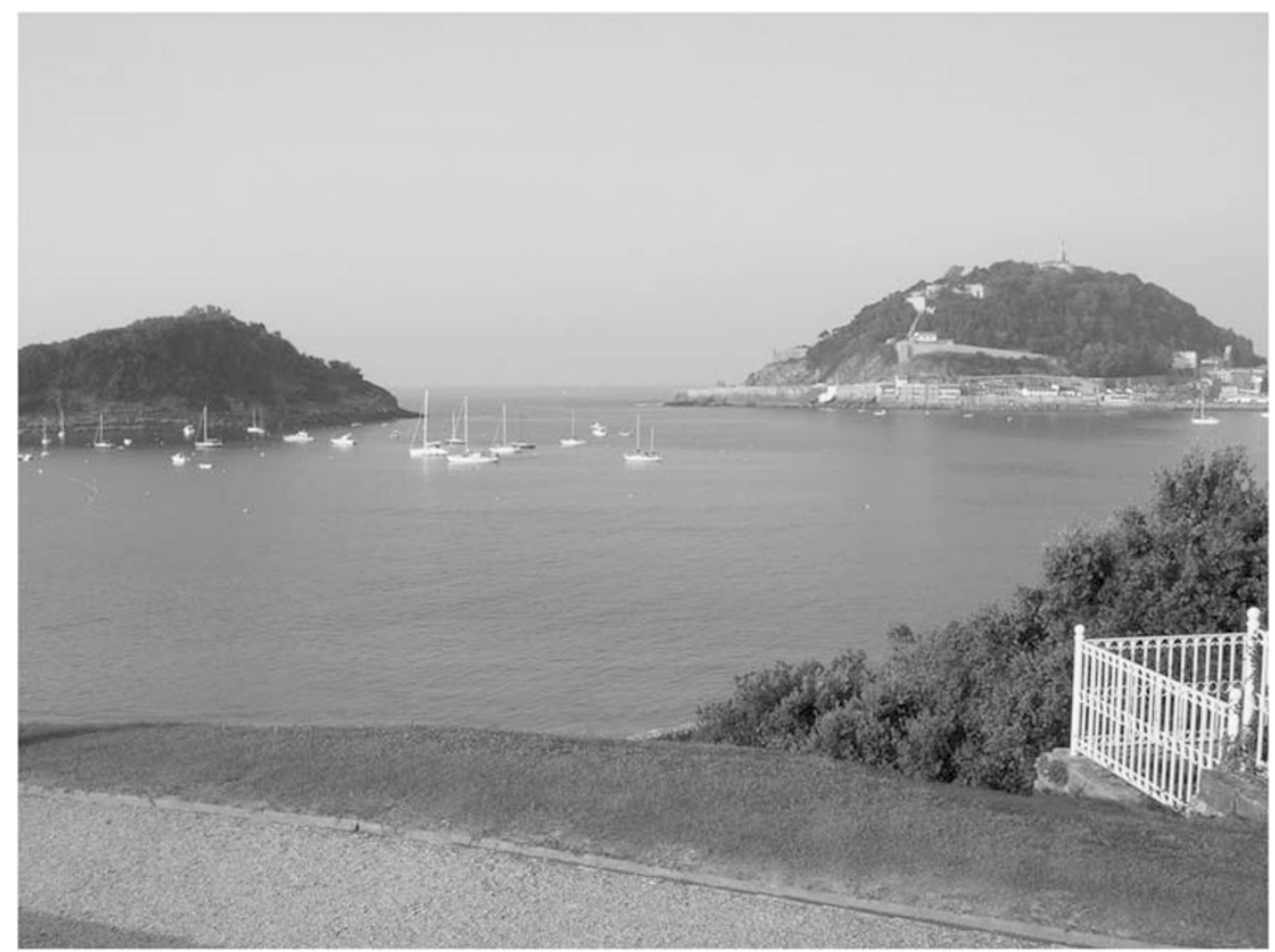

Figure 3 LaConcha Bay, Île de Santa Clara and Mont Urgull. 
proline derivatives as antagonists of leukocyte -function-associated antigen-1 (LFA-1) that showed antiproliferative, antimetastatic and antiadhesive properties in certain cancer cells. Some compounds were found to inhibit the interactions of LFA-1 with ICAM-1 or VLA-4 with VCAM-1 and show antimetastatic activity in mice. Gaëlle Malik (University of Bordeaux) described the synthesis of vescalin, a DNA topoisomerase II inhibitor more potent than etoposide, by using an intramolecular biomimetic coupling with chloranil and an aminecopper complex followed by a C-arylglycosidation.

In another lecture on the theme of biosynthesis, Professor Tadhg Begley (Texas A \& M University) reported recent results from his continuing studies of thiamin biosynthesis in which the biosynthetic pathway in yeast differs from that in bacteria. ${ }^{13}$ Thi4 is a single enzyme that assembles the thiazole component from glycine, cysteine and a pentose moiety, but efforts to reconstitute biosynthesis from overexpressed protein unexpectedly failed. It appears that the enzyme contains two nearby thiol groups and contributes $\mathrm{H}_{2} \mathrm{~S}$ with simultaneous formation of a lantibiotic-type sulfur bridge in a one-to-one substrate-to-enzyme manner, and the active enzyme is not regenerated after contributing the sulfur, so the enzyme turnover was unexpectedly one.

The conference ended with an excellent evening gathering at the Restaurante Monte Igeldo overlooking the city and bay.

The next conference in this series, ICCA-12, was announced for September, 2011, in Berlin under the organization of Professors Kalesse, Kirschning and Süssmuth.

\section{ACKNOWLEDGEMENTS}

I thank Professor Jesus Aizpurua, the conference organizer, for reviewing the paper.

1 Sun, C. et al. A robust platform for the synthesis of new tetracycline antibiotics. J. Am. Chem. Soc. 130, 17913-17927 (2008).

2 Gordo, S. \& Giralt, E. Knitting and untying the protein network: modulation of protein ensembles as a therapeutic strategy. Protein Sci. 18, 481-493 (2009).

3 Ishikawa, H., Suzuki, T. \& Hayashi, Y. High-yielding synthesis of the anti-influenza neuramidase inhibitor (-)-oseltamivir by three 'one-pot' operations. Angew. Chem. Int. Ed. 48, 1304-1307 (2009).

4 Mandity, I. M. et al. Design of peptide foldamer helices: a stereochemical patterning approach. Angew. Chem. Int. Ed. 48, 2171-2175 (2009).

5 http://www.weizmann.ac.il/sb/faculty_pages/Yonath/home.html.

6 Wekselman, I. et al. Ribosome's mode of function: myths, facts and recent results. J. Peptide Sci. 15, 122-130 (2009).

7 Demain, A. L. \& Sanchez, S. Microbial drug discovery: 80 years of progress. J. Antibiot. 62, 5-16 (2009).

8 Baker, D. D., Chu, M., Oza, U. \& Rajgarhia, V. The value of natural products to future pharmaceutical discovery. Nat. Prod. Rep. 24, 1225-1244 (2007).

9 Kirschning, A., Taft, F. \& Knobloch, T. Total synthesis approaches to natural product derivatives based on the combination of chemical synthesis and metabolic engineering. Org. Biomol. Chem. 5, 3245-3259 (2007).

10 Von Nussbaum, F., Brands, M., Hinzen, B., Weigand, S. \& Habich, D. Antibacterial natural products in medicinal chemistry - exodus or revival? Angew. Chem. Int. Ed. 45, 5072-5129 (2006).

11 Dixon, N., Wong, L. S., Geerlings, T. H. \& Micklefield, J. Cellular targets of natural products. Nat. Prod. Rep. 24, 1288-1310 (2007).

12 Lange, R. P., Locher, H. H., Wyss, P. C. \& Then, R. L. The targets of currently used antibacterial agents: lessons for drug discovery. Curr. Pharmaceut. Design 13, 3140-3154 (2007).

13 Jurgenson, C. T., Begley, T. P. \& Ealick, S. E The structural and biochemical foundations of thiamin biosynthesis. Annu. Rev. Biochem. 78, 569-603 (2009). 